\title{
Molecular genetic methods for assessing drought resistance of spring barley
}

\author{
Novikova A.A., Bogdanova O.V.* \\ Federal Research Centre of Biological Systems and Agrotechnologies of Russian Academy of Sciences, \\ Orenburg, Russia \\ *email: olga_bogdanova_1995@mail.ru
}

The creation of varieties with high adaptive potential is an important condition for the formation of highly productive agroecotypes. In the conditions of sharply continental climate of the Southern Urals, the lack of moisture during the growing season is a limiting factor in realization of the potential yield of agricultural crops. In the breeding process, the assessment of the drought resistance of the variety is based on long-term observations in the field. The introduction of molecular genetic methods for assessing plant material will eliminate dependence on weather conditions, shorten the research time and increase the efficiency of selection.

As an object of research, we took varieties and lines of spring barley from the control nursery of the Department of Breeding and Seed Production of Grain Crops of the Federal State Budgetary Research Institution FSC BST RAS. For research, a working sample was formed, it included 150 samples with high potential productivity. The spring barley variety Natali was used as a control. The prevailing conditions of weather factors served as a good background for assessing the material for its resistance to air and soil droughts (the amount of precipitation was more than 2 times lower than the average long-term values). Phenotypic assessment and assessment of productivity indicators of the stem showed that the numbers have a wide norm of reaction to stress caused by a lack of moisture.

The second stage of the research includes the formulation of a methodology and assessment of drought tolerance of cultivars by the method of quantitative assessment of cDNA genes associated with the response of plants to water stress. Total RNA is planned to be isolated from etiolated seedlings using ready-made kits according to the manufacturer's instructions.

In conclusion, the assessment of the genetic potential of the working sample, the degree of phenotypic manifestation of the studied genes will be carried out, and donors of economically valuable traits will be selected. 